\title{
Software Defined Networks: Review and Architecture
}

\author{
Tanweer Alam 1 , Mohammed Aljohani ${ }^{2}$ \\ Faculty of Computer and Information Systems \\ Islamic University of Madinah \\ Saudi Arabia \\ e-mail: tanweer03@gmail.com
}

(APA style, Justify, Arial 10pt) Example:

To cite this document:

Alam, T., \& Aljohani, M. (2020). Software Defined Networks: Review and Architecture. IAIC Transactions on Sustainable Digital Innovation (ITSDI), 1(2), 143-151. https://doi.org/https://doi.org/10.34306/itsdi.v1i2.114

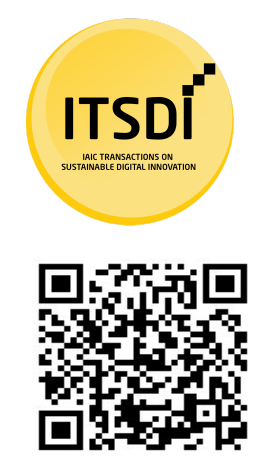

Author Notification 23 Maret 2020

Final Revised 24 Maret 2020 Published 25 Maret 2020

\begin{abstract}
In the fifth-generation communication system, secured and reliable data packets will rely on the network with high availability and low latency. The 5G network enables the dynamic control of nodes with low latency. Ultra-reliability is one of the challenging tasks in software-defined networks. The ultra-reliability feature is an interface working with high availability and low latency that brings in fifth-generation networks. The Internet of Things will work in the societies so that it required ultra-reliability features to empower the peoples as well as machines for collaborating with their neSoftware Defined Networksighbors. The connections among ultra-reliability networks are extremely hard situations to keep low packets corruption. The Software Defined Networks (SDN) will provide a platform for measuring, controlling, monitoring and scaling the smart objects in reality or virtually. Virtualization enables IT, specialists, to combine or separate the considerable networking or create new programmable networking among virtual machines. In this article, the authors are reviewed the architecture of SDN based on virtualization under the umbrella of $5 G$ networks.
\end{abstract}

Keywords: Ultra-Reliability; Internet of Things; 5G Networking; Software Defined Networks; Virtualization; Security.

\section{Introduction}

The Virtualization enables information technology specialists to combine several physically created networks or separate considerable networking into several parts or form new software-defined networking among virtual machines. A few years ago, virtualization has expanded in the world especially in many data centers for example in server consolidation, server security, and clouds. This easily happened due to an increase in hardware performance (Figure 1). Reducing the costs of the data centers, Virtualization technology can support the maximum usability of hardware devices on the physical server [1].

By using virtualization technology, executing many operating systems and several applications on a single server is the approachable goal. This goal is useful to improve the performance of networks, increase automatic technology and easily manage the networks using programs. Virtualization technology has been known a long time ago, on the time of mainframes from 1960 to 1970s. The definition of virtualization has reformed a lot since it started. The definition was that allocating mainframes to allow for multiple applications to process concurrently [2]. Systems could only run a solo application simultaneously. 


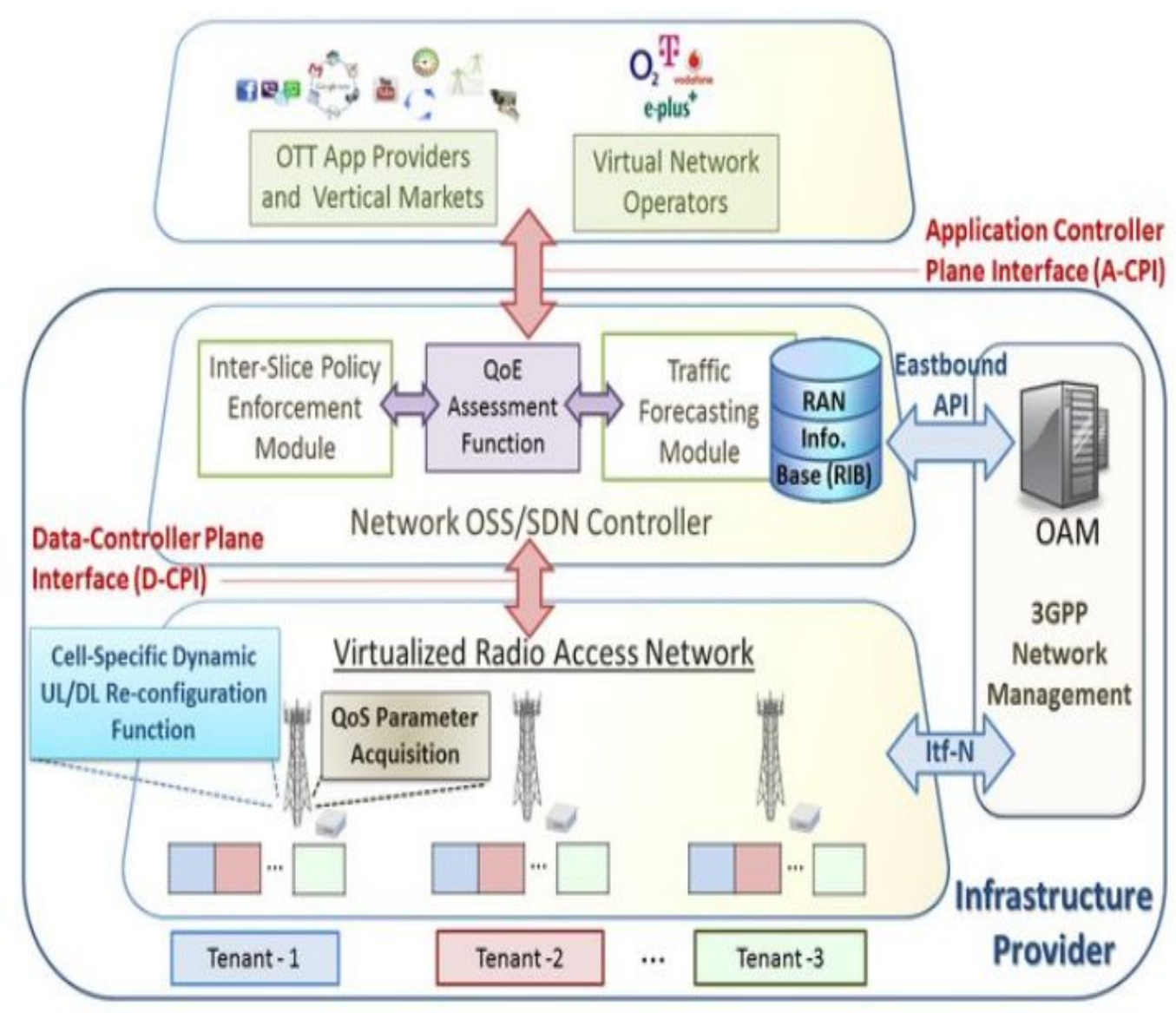

Fig. 1: Virtual Infrastructure [2]

Many organizations found it costly to purchase mainframes doing one task. From that time virtual machines, IBM mainframes have developed. In 1990 the x86 base's systems were released and the embracing from operating systems such as Linux and Windows have moved virtualization from mainframes based applications to client-server distributed based applications. It is an easy approach now to run different applications simultaneously not like mainframes. In the early 2000 s virtualization technology starts to advance and become more reliable on the $x 86$ base's systems. Data centers become more expensive to run with many devices and applications.

Virtualization has been an indispensable role with emerge of distributed computed systems. Clients were given virtual resources from multi or single shared physical devices. The idea of virtualization helped clients to use a large pool of shared resources with excellent control and customization. The combination of virtualization concepts and networking is the main center of software-defined networking. Virtualization is the optimal answer for the utilization of physical resources. The virtual networks are categorized into inner and outer virtual networks. The inner virtual networks enable virtual machines to exchange information within the range of servers. The outer virtual networks allow switches, adapters and local area networks outside the range of servers [4].

\section{Software-Defined Networking}


Software-defined networking is a new technology that will use in the future to replace the current network with a low latency network. From 1999, we are using networking based on switches, routers and hubs etc., the new versions are coming but the technology is the same. In 2005, VOIP introduced and deployed, at that time some programs implemented for voice and video calling ex. Skype. Until now we are using the same technology with the advanced version. SDN provides us the high-speed real-time connectivity with low latency. SDN is a new interface that is real-time, optimized, effective, flexible, measurable and easy to adapt with a high bandwidth (Figure 2). SDN is designed to separate the control plane and forwarding plane to enable the network control to become programmable that delivers decisions according to the specific network requirement. The software-defined networking has three layers- Application Layer, Control Layer and forwarding layer [3].

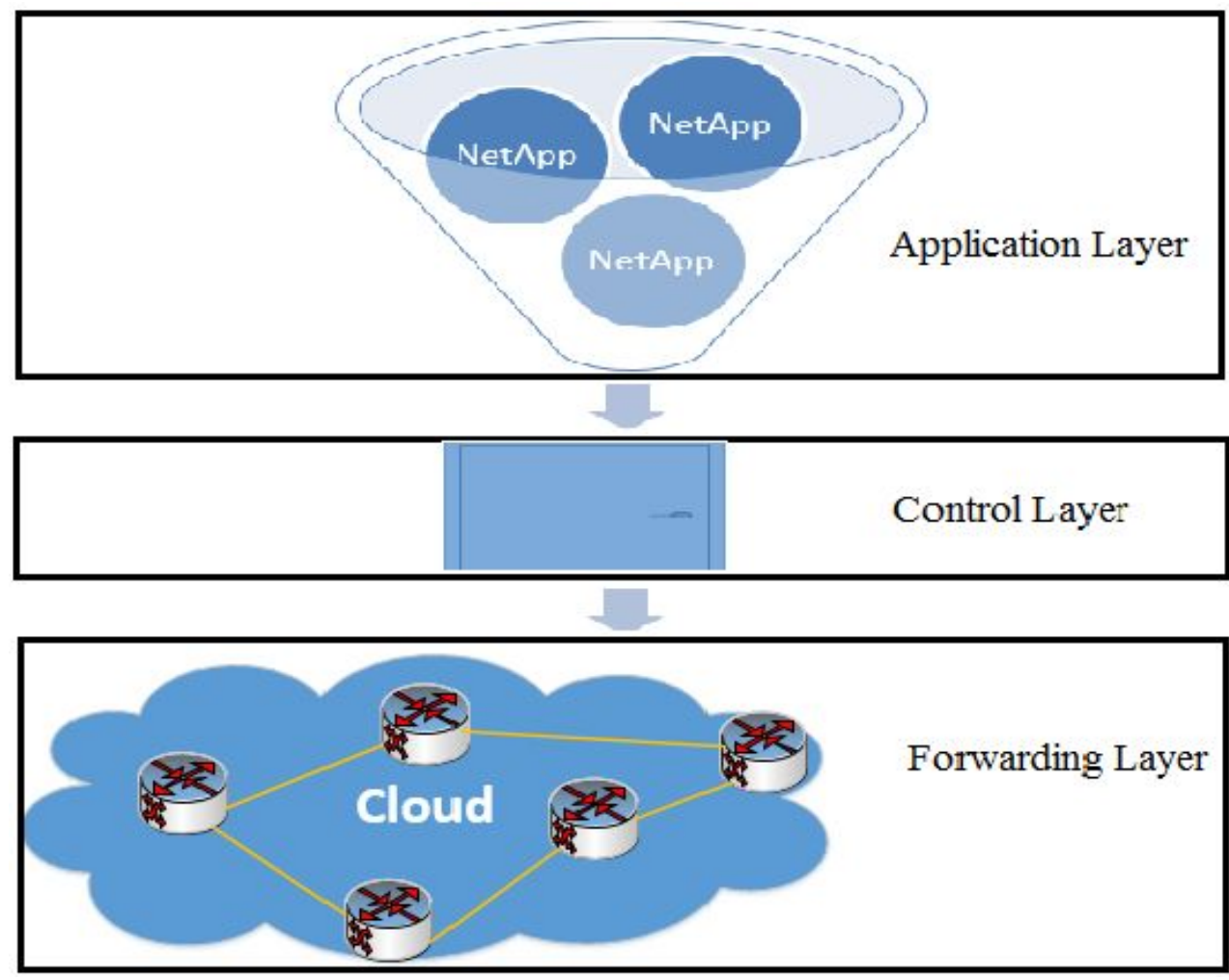

Fig. 2: SDN layers Architecture

The SDN control plane works as the mastermind of networking. SDN is the intelligent technology that takes snapshots of the entire networks and their routing tables for instruct the forwarding plane to forward the network traffic to switches and routers. There are two kinds of APIs in SDN, the first is called South Bound API (SBAPI) and the second API is called North Bound API (NBAPI). It is an interface between the forwarding plane and the control plane (Figure 3). The SBAPI is used to insert information to the switches and routers and NBAPI inserts the information to the applications. It is an interface between network applications and the control plane [4]. 


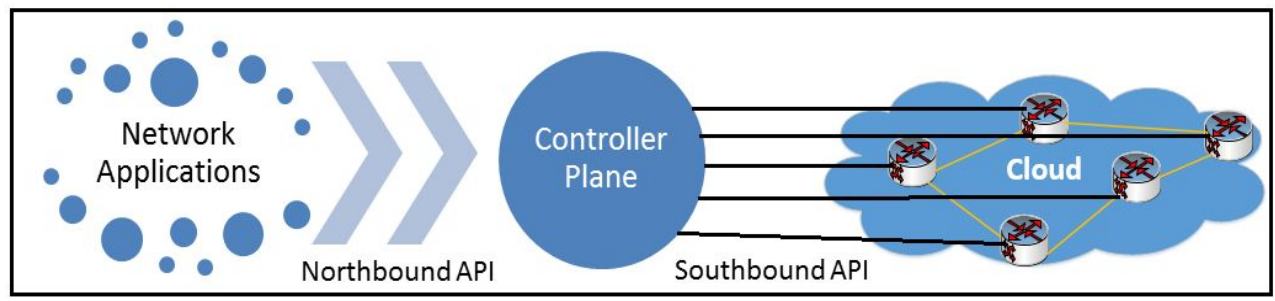

Fig. 3: Northbound and Southbound APIs

SDN architecture has three phases. The first phase is the forwarding plane, it is used to forward information down to the switches and routers. The second phase is the control plane; it is used to push the information to the application. The third phase is the Network Applications, it is used to provide service directly by the program. The SDN architecture has the following key elements-

i) Direct Programming Approach: SDN has the directly programmable approach to separate the control plane and forwarding plane.

ii) Agile Approach- It is used to move quickly and easily to forward the network traffic according to the specific requirements.

iii) Centralized Approach- It is used to centralize the entire network using programmable SDN controllers.

iv) Programmable Configuration Approach- the SDN manages the entire network securely and optimized easily and quickly in real-time using the automatic execution of SDN programs.

Software-defined networking has the following elements.

1. Decoupling of Control plane- in this element the open flow switch improves the specifications of Cookies (version 1.0) for identification of forwarding information and control the routing table (version 1.4) for discovering the path.

2. Logical Centralization- This element monitors the traffic and data packets that are shared by applying the requests. Also, it filters all events that are fired by multi controllers.

3. Northbound APIs- In Northbound APIs, the QoS per network application improved using the priority of the queue per port as well as per flow meters.

4. Programmability- SDN is a decoupled technology that is completely programmable. It reuses the code in groups of headers and routing tables.

5. Flow Entries- This element improves the several tables of routing to discover the path of the network.

The decoupling of the control plane represents the decisions for handling the traffic efficiently and quickly using the centralized controller of SDN.

\section{Hypervisor}

The word "hypervisor" originates from the word supervisor, which now means the operating system, and the inventor of the hypervisor adds hypervisor with more power more than the supervisor. The hypervisor is recognized as a Virtual Machine Monitor (VMM) which software supports virtualization. The hypervisor is accountable for operating virtual machines such as creating, halting, and monitoring (Figure 4). Hypervisor administers all guest systems and sorts all the resources they require for example central processing unit (CPU) random access memory (RAM), network interface card (NIC) and Hard-disk storage. The hypervisor is divided into two categories [12]. 


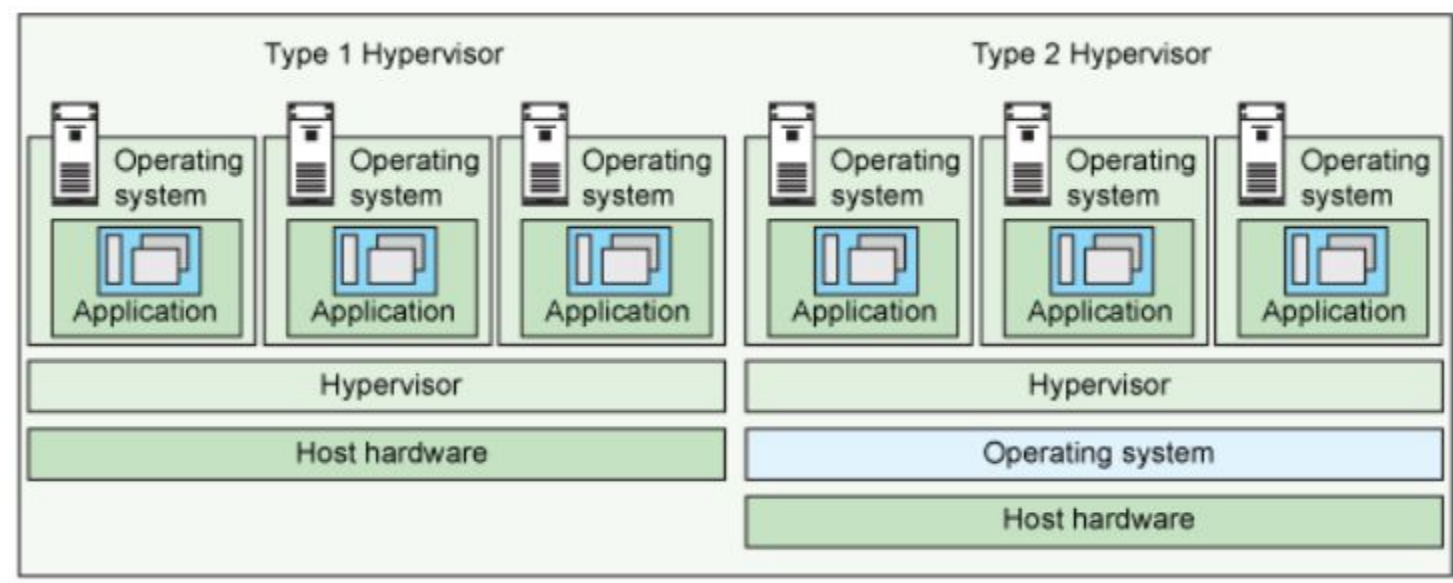

Fig. 4: Hypervisor [12]

The first type is titled as the bare-metal hypervisor. Type 1 hypervisor is working directly on physical hardware. It is accountable for all guest system's needs from physical Hardwar. Type 1 hypervisor is commonly used in the server virtualization environment. This kind of supervisor has fewer drivers to be instilled Dome-0 is identified to give guest virtual machine power to monitor and control the hypervisor. In order to function Type 2 hypervisor needs all host operating systems. The hypervisor will be instilled at the top of the operating system. This approach has some benefits it has a fewer driver issues due to the communicating between OS and hardware is more reliable for communicating hypervisor and hardware. The disadvantage of type 2 is the lack of performance compared to the type1. We refer to the Guest is where the operating system runs on the top of the hypervisor. The guest environment is a complete virtualized from Operating Systems to all the resources requirements for the guest. Sometimes driver's installation is needed to run guest operating systems [6].

\section{Why Virtualization?}

For applications use Virtualization has more benefits comparing to other old-style infrastructure. For server utilization and consolidation, virtualization is the ideal solution to deploy many technologies. Virtualization is more cost-effective to run and manage. Virtualization technology is very useful for testing products using different environment without buying many products. At the time of maintenance or the time of disaster it east to allocate virtual machines to other places until the cause goes and return back with less downtime for the system. This technology can play a big role in the recovery plan at the time of a security attack. In addition, there is some issue with virtualization such as some application running virtual machines needs high resource utilization does not perform fine on the system. This issue minimizes the overall performance of the virtualization environment [7].

\section{Infrastructure as a Service}

Infrastructure as a Service (laaS) is a term comes from virtualization usability when some provide built their own cloud virtual environment on their data centers and start rent these services to organizations. This approach removes many obstacles in the world of IT industry such as the cost of physical hardware maintenance, housing space, and powering implications laas allows the organization to reduce cost. laaS has some weaknesses in terms of security and privacy. laaS providers play an immense part to secure important data that leave the organization to be stored on the cloud [8].

\section{Virtual Memory Operation}

When the end-user wants to run a large program that is impossible to fit in physical 
RAM memory .the program is divided into same size chunks, which are called "pages" size in $4 \mathrm{k}$ to $64 \mathrm{k}$ in usual. In addition, the memory address is divided into Logical pages, which has the same size. When a process wants, execute a memory address the memory monitor searches from hard disk about page address to allocate in empty space in the RAM fetching from in physical address to another based on the processing of the instructions of program pages subsequently. When a computer does not have an available physical page then it is time to move to a virtual page through a sweeping technique .memory monitor would play the role of swiping of the physical page into the hard disk and knows which pages belong to a different process (Figure 5).

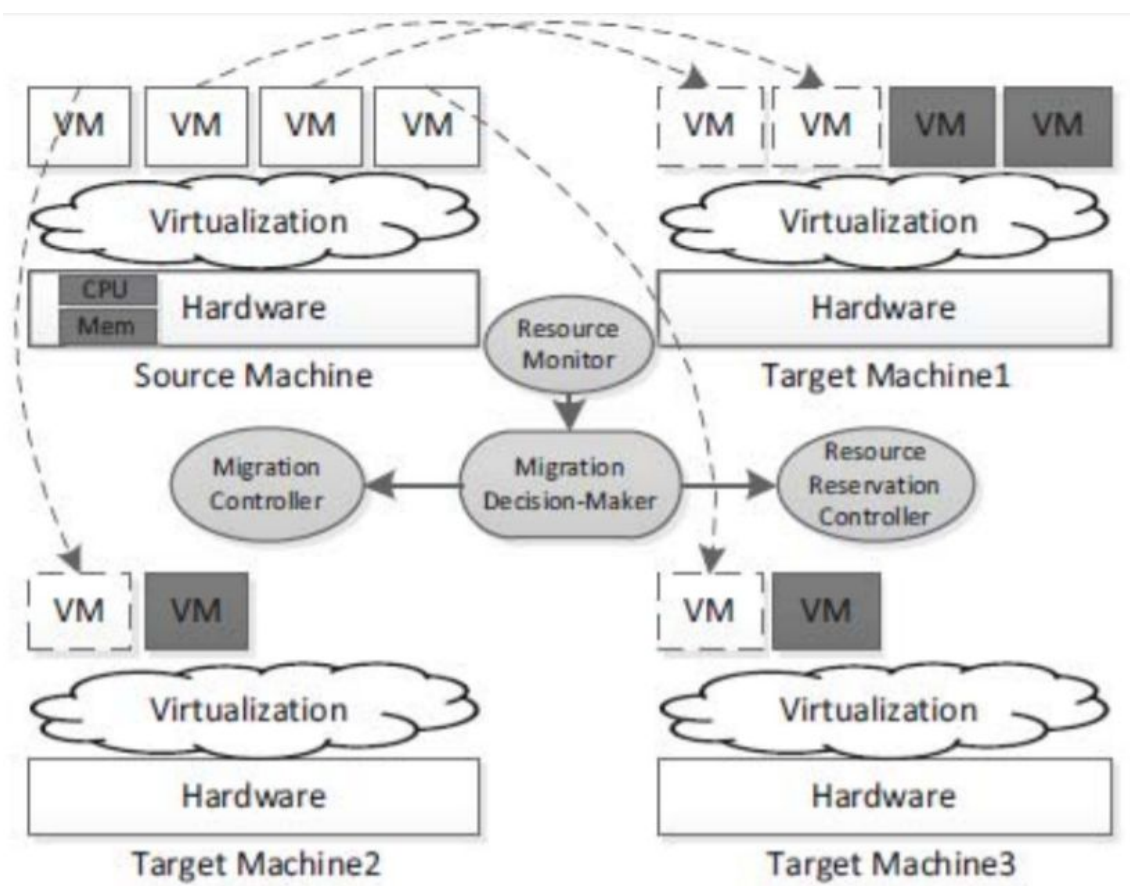

Fig. 5: Virtual Machine Memory mapping [14]

Computer main processer can see all the memory address space as one block address space through mapping technic .operating system OS take the role of creating virtual address spaces and connect real memory address locations to virtual address locations, the operating system OS has the ability to make virtual memory address space which is bigger than actual of physical memory address built in the computer machine the memory management unit (MMU) is responsible for the monitoring of virtual memory in the computer hardware [9]. This piece of hardware would convert the virtual address by processing it in a physical address. A virtual memory system makes it easy to run multiples applications on the same computer and acts as a whole memory on the process of one program by translating virtual memory addresses to physical memory address mechanisms [10].

\section{Virtual and Physical Memory Mapping}

The main concept of virtual memory system is to establish a process for the computer to use virtual address to run a program while the operating system would be responsible of mapping physical address space of the computer and virtual address space of application .each program sored in the memory with its data it named a process. Every process is 
allocating with address space an address space is can be stored as sequence of instructions of codes and variables inside the memory and can be used to execution .os can arrange the size of address space to program which is not usually fixed but allocating based on the need of program while it's in processing time .then additional memory would be given from os when it's needed (Figure 6). There are three ways of how the process runs first is loading the instruction, storing instruction and fetching instruction for the program. These steps created for virtual address space then it will be mapped onto the physical address.in addition, computer using virtual memory will handle the translating between virtual memory to physical memory mapping all the time special hardware will make this possible and easy task to execute. There are six steps in order to explain the transition between virtual memory and physical memory [11].

Step A: See of internal table virtual memory for the process. Checking if it has a reference or not.

Step B: if virtual memory was not stopped the process. If page is not stored then the page will be requested.

Step C: search for any available frame in the Ram.

Step D: organizing disk process to see wanted page into RAM allocation.

Step E: when the disk is finished from reading, change the internal table to keep with the process.

Step F: recycle the process for program the process can see the Page as it is always in the memory.

Virtual memory system increased security because applications do not play any role in memory management and the isolation of the memory.

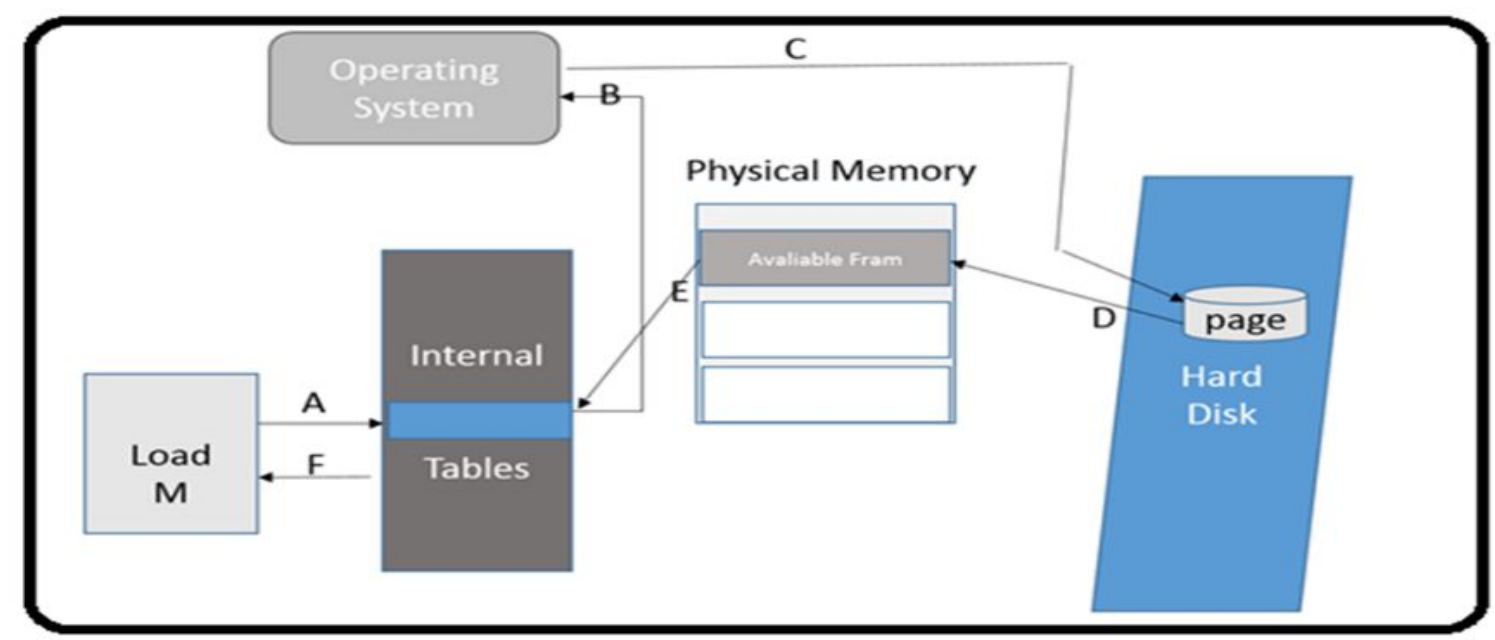

Fig. 6: Virtual Memory Mapped Physical Memory

\section{Server Virtualization}

After the successful approach of visualizing computer memory and the advantages, were given to the end-users computer engineers were ready to achieve another successful approach: how to give many users the ability to use a single computer at the same time. The answer to this issue is to virtualize the entire system. Each end-users were given a virtual machine as a subsystem to control. The operating system was called supervisor which operate all physical task on the system .a software called hypervisor was created to be more superior than the operating system. Hypervisor takes the role of operating system 
and manages all virtual machines given to the users.

\section{Conclusion}

Software-defined networking (SDN) technological progress has become an access control strategy that allows dynamic, programmatic effective network setup to enhance network performance and monitoring, making it more like cloud computing than conventional network management. The SDN aims to acknowledge the fact that perhaps the static structure of conventional networks is decentralized and complex. The SDN aims to centralize network expertise in a single network element by disassociating the network packet filtering process (data plane) from the forwarding method.

\section{References}

[1] Li, Yong, and Min Chen. "Software-defined network function virtualization: A survey." IEEE Access 3 (2015): 2542-2553.

[2] Costanzo, Salvatore, Rudraksh Shrivastava, Konstantinos Sarndanis, Dionysis Xenakis, Xavier Costa-Pérez, and David Grace. "Service-oriented resource virtualization for evolving TDD networks towards 5G." In 2016 IEEE Wireless Communications and Networking Conference, pp. 1-6. IEEE, 2016.

[3] Porras, P.A., Cheung, S., Fong, M.W., Skinner, K. and Yegneswaran, V., 2015, February. Securing the Software Defined Network Control Layer. In NDSS.

[4] Alam, Tanweer. "Fuzzy control based mobility framework for evaluating mobility models in MANET of smart devices." ARPN Journal of Engineering and Applied Sciences 12, no. 15 (2017): 4526-4538.

[5] Alam, Tanweer. "Middleware Implementation in Cloud-MANET Mobility Model for the Internet of Smart Devices", International Journal of Computer Science and Network Security, 17(5), 2017. Pp. 86-94

[6] Alam T, Benaida M. CICS: Cloud-Internet Communication Security Framework for the Internet of Smart Devices. International Journal of Interactive Mobile Technologies (iJIM). 2018 Nov 1;12(6):74-84. DOI: https://doi.org/10.3991/ijim.v12i6.6776

[7] Tanweer Alam, Baha Rababah, "Convergence of MANET in Communication among Smart Devices in loT", International Journal of Wireless and Microwave Technologies (IJWMT), Vol.9, No.2, pp. 1-10, 2019. DOI: 10.5815/ijwmt.2019.02.01

[8] Tanweer Alam, "loT-Fog: A Communication Framework using Blockchain in the Internet of Things", International Journal of Recent Technology and Engineering (IJRTE), Volume-7, Issue-6, 2019.

[9] Tanweer Alam, "Blockchain and its Role in the Internet of Things (IoT)", International Journal of Scientific Research in Computer Science, Engineering and Information Technology, vol. 5(1), pp. 151-157, 2019. DOI: https://doi.org/10.32628/CSEIT195137

[10] Alam, Tanweer. (2018) "A reliable framework for communication on the internet of smart devices using IEEE 802.15.4." ARPN Journal of Engineering and Applied Sciences 13(10), 3378-3387.

[11] Tanweer Alam, "A Reliable Communication Framework and Its Use in the Internet of Things (loT)", International Journal of Scientific Research in Computer Science, Engineering and Information Technology (IJSRCSEIT), Volume 3, Issue 5, pp.450-456, May-June.2018 URL: http://ijsrcseit.com/CSEIT1835111.

[12] Bahadur, Nitin, and Kenneth E. Gray. "Software defined network controller." U.S. Patent 9,450,817, issued September 20, 2016.

[13] https://vapour-apps.com/what-is-hypervisor/

[14] Deshmukh, Pranjali P., and S. Y. Amdani. "Survey of memory streaming techniques for virtual machine in cloud environment." In 2017 International Conference on Energy, Communication, Data Analytics and Soft Computing (ICECDS), pp. 1198-1200. IEEE, 2017. 
[15] Tanweer Alam, Mohamed Benaida, "Blockchain, Fog and IoT Integrated Framework: Review, Architecture and Evaluation”, Technology Reports of Kansai University, Volume 62 , Issue 02, 2020.

[16] Alam, T., 2020. Cloud Computing and its role in the Information Technology. IAIC Transactions on Sustainable Digital Innovation (ITSDI), 1(2), pp.108-115. 